\title{
Towards multiple 3D bone surface identification and reconstruction using few 2D X-ray images for intraoperative applications
}

\author{
Simant Prakoonwit \\ Department of Computer Science and Technology, University of Bedfordshire, UK \\ Email: Simant.Prakoonwit@ieee.org,Simant.Prakoonwit@beds.ac.uk
}

\begin{abstract}
This article discusses a possible method to use a small number, e.g. 5, of conventional 2D X-ray images to reconstruct multiple 3D bone surfaces intraoperatively. Each bone's edge contours in X-ray images are automatically identified. Sparse 3D landmark points of each bone are automatically reconstructed by pairing the 2D X-ray images. The reconstructed landmark point distribution on a surface is approximately optimal covering main characteristics of the surface. A statistical shape model, dense point distribution model (DPDM), is then used to fit the reconstructed optimal landmarks vertices to reconstruct a full surface of each bone separately. The reconstructed surfaces can then be visualised and manipulated by surgeons or used by surgical robotic systems.
\end{abstract}

\section{INTRODUCTION}

The ability to be able to access and update 3D patient-specific surface models of the bones and other structures during an operation is very valuable. It can augment the surgeon's view of the objects of interest and help with navigation, measurement and surgical planning. The 3D model can also be used with a surgical robot to accurately carry out surgical procedures on an up-to-date models of the structure of interest. However, a 3D reconstruction of bones and other structures during an operation is a nontrivial task. Most of existing technologies are not feasible to be implemented in an intraoperative environment. Normally, a 3D reconstruction has to be done by a CT or an MRI pre operation or post operation. Due to some physical constraints, it is not feasible to utilise such machine intraoperatively. The models have to be reconstructed before or after an operation. If the model is required to be updated during an operation, the patient has to be moved from the operating table to a CT or MRI machine before returning to the operating table again. A special type of MRI has been developed to overcome the problem. However, all normal surgical tools and instruments cannot be employed. This seriously limits the use in orthopaedic applications. Also any metal surgical robots cannot be utilised. In addition, they are expensive and/or induce high radiation doses (Rajamani, Styner et al. 2007). The costs associated with CT are MRI techniques are high. CT imaging can also produce radiation hazards.

The main aim of this article is to propose a new design and possible method towards rapidly identifying, reconstructing and updating patient-specific 3D surface models of multiple bones using a small number of 2D conventional X-ray images feasible for intraoperative applications. The technique should be capable of reconstructing 3D bone surface models without having to move patient from the 
operating table. This article is based on our preliminary work (Prakoonwit 2011) which can only deal with a single object. New effective methods on edge contour detection and multiple object identification are presented in this work.

\section{BACKGROUND}

In an intraoperative environment where the scanning geometry of a CT or MRI is not suitable, a Carm conventional X-ray system can be used to acquire a number of 2D images to reconstruct a full 3D volumetric description, in terms of voxels, of an object of interest, e.g. (Atesok, Finkelstein et al. 2007; Ritter, Orman et al. 2007; Zbijewski and Stayman 2007). However, to reconstruct at a reasonable resolution, the number of 2D images required is very high, e.g. 40 to 180 images, and to extract the surface of an object from the reconstructed voxels is very computationally expensive. Moreover, due to the large number of 2D X-ray images required, the patient is inevitably subjected to high dose of radiation.

Another approach is to use statistical shape analysis and modelling, e.g. (Cootes, Taylor et al. 1995; Dryden and Mardia 1998; Rajamani, Styner et al. 2007; Zheng, Dong et al. 2007; Prakoonwit 2011) and (Zhu and Li 2011), which has been an important tool in 3D model reconstruction from incomplete data. In this approach, only a small number of sparse landmark vertices on the surface of an object, e.g. a bone, are needed to be determined. Those sparse landmark vertices alone contain inadequate information for the complete 3D surface reconstruction of an object. Hence, a priori knowledge is required. A statistical model can be reconstructed from a set of training surfaces representing reasonable variations of the surfaces of an object of interest. In intraoperative applications, the statistical model is then used as prior knowledge in the reconstruction process to fit to the patient anatomy using intraoperatively acquired sparse landmark vertices. Thus, in conclusion, the aim of statistical shape model fitting is to extrapolate from an extremely sparse and incomplete set of 3D landmark vertices to a complete and reasonably accurate 3D anatomical surface. The fitting process aligns and deforms the statistical shape model to fit the sparse landmark vertices. Therefore the model-based approach is widely accepted due to their ability to effectively represent objects (Morooka, Nakamoto et al. 2013).

Many researchers have presented methods for fitting statistical models to sparse vertices to create full patient-specific surfaces. The input data can be acquired from a small number of 2D X-ray images (Fleute and Lavallee 1999; benameur, Mignotte et al. 2003; Benameur, Mignotte et al. 2005; Lamecker, Wenckeback et al. 2006) or digitised sparse vertices (Fleute and Lavallee 1998; Fleute, Lavallee et al. 1999; Rajamani, Styner et al. 2004; Rajamani, Ballester et al. 2005). Principle Component Analysis (PCA) based statistical shape models have been widely used in surface extrapolation. In (Fleute, Lavallee et al. 1999), a joint optimisation technique is applied to fit the statistical model to intraoperatively digitised landmark vertices. In this method both pose and deformation are concurrently optimized. Later, Chan et al (Chan, Edwards et al. 2003), used a different approach by optimising deformation and pose separately and implementing the iterative closest point (ICP) method. Rajamani, et al (Rajamani, Styner et al. 2007), developed a new technique using a Mahalanobis distance weighted least square fit of the deformable model to the landmark vertices. The surface model of a proximal femur was reconstructed in (Zheng, Dong et al. 2007) by using a Dense-Point Distribution Model (DPDM) and employing two-stage deformation processes: statistical instantiation, which stably instatiates a surface model from the DPDM using a statistical approach; kernel-based deformation, which further refines the surface model. Two-view or multi-view 2D X-ray images have also been used with statistical shape models to reconstruct femur and pelvis in (Lamecker, Wenckeback et al. 2006; Zheng, Ballester et al. 2006; Kurazume, Kakamura et al. 2009; 
Zheng, Gollmer et al. 2009). Pelvis and vertebra bones can also be reconstructed from uncalibrated Xray images as shown in (Zheng 2009; Zheng 2010; Zheng, Nolte et al. 2011). 3D models for Femur kinematics studies can also be reconstructed from biplane fluoroscopy (Baka, Bruijne et al. 2012). Those methods rely mostly on the information from 2D X-ray images without sparse 3D points on the surface of the objects. Hence, some surface information for the 3D reconstruction is not complete.

The existing methods using sparse 3D points mentioned earlier are capable of producing 3D surface models. However, the performance of the algorithms and the accuracy of the resulting models very much depend on how well the input sparse landmark vertices are obtained and how well they can represent the important features of the surface. The existing methods of creating the input sparse landmark vertices are not fully automatic and the landmark vertices are not optimally distributed on the surfaces resulting in some important features may not be well captured or represented. In this article, a method is proposed to solve this problem. The main contributions of this work, therefore, are: a new design of scanning geometry; a novel automatic, without user intervention, method for determining 3D landmark vertices which are optimally distributed on a surface and multiple object identification. Some proof of concept computational experiments have been conducted to reconstruct a femur bone and a set of knee joint bones. The results show that the proposed method is capable of reconstructing an acceptable 3D surface.

\section{PROPOSED METHOD}

In our proposed method, a small number, e.g. 5, of X-ray images are taken at appropriate projection angles. Edges contours are then extracted and identified for each bone in the scene. The 3D landmark vertices are determined by paring all the edges in all images. Once the landmark vertices are found, a statistical shape model is employed to fit the landmarks to create a full 3D surface model.

\subsection{Edge detection and representation}

Each X-ray image is enhanced prior to edge detection to reduce the noise level, sharpen the edges and to enhance the contrast of the edges. Techniques such as adaptive smoothing and adaptive histogram equalization can be used to enhance the image. In this research, the grayscale enhancement method described in (Abidi, Mitckes et al. 2003) has been adopted. First, linear regression is applied to stretch the pixel range within the given image so that the maximum and minimum pixel values cover wider range. Then gamma intensity adjustment is performed followed by histogram equalization. Those procedures require manual parameter adjustment. However, a set of common parameters can be used for most of the X-ray images in the application.

In our preliminary work (Prakoonwit 2011), the Gradient edge detector with relaxation labeling (Matalas 1996) to reinforce contours and suppressing invalid edges was used. To improve the quality of edge contours of bones in X-ray images, a new biologically-inspired contour detection method (Kang 2011) is therefore adopted.

The method is based on human vision in perceiving of contours in images. According to (Westheimer 1979), human vision is 5 to 10 times more perceptive to positions or phases than to magnitude differences in an image. Phase distortion reduces the ability of human visual system to recognise objects or contours in an image than intensity distortion. Edge contours in images can be interpreted as the points of maximum phase congruency in frequency domain (Morrone, Ross et al. 1986) which, to a large extend, correspond with edge contour features perceived by human visual system. By combining the biological findings with Gestalt theory (Desolneux, Moisan et al. 2008), PCTV method was developed (Kang 2011). The method can be used to detect edge contours in X-ray images with superior quality compared to other methods such as Canny edge detector (Canny 1986), SUSAN detector (Smith and Brady 1997), the compass operator (Ruzon and Tomasi 2001) and phase 
congruency (PC) method (Kovesi 1999) as demonstrated in (Kang 2011). Also edge contours detected by PCTV are of higher quality than those derived by the edge detection method in (Matalas 1996).

To reconstruct landmark vertices, the most effective way is to represent edge contours analytically. Therefore, those edge contours are fitted with cubic B-spline curves. Each edge contour $c$ is represented by a sequence of 3rd order parametric curve segments. To detect edge contours and conduct B-spline curve fitting, we adopt the techniques described in (Matalas 1996).

\subsection{Landmark point reconstruction}

In this section, we consider how multiple 3D landmark points on a 3D surface can be found by pairing 2D X-ray images, using the characteristics of epipolar geometry. The method is based on our previous work (Prakoonwit and Benjamin 2007). In contrast to current methods, e.g. (Rajamani, Ballester et al. 2005; Zheng, Dong et al. 2006; Rajamani, Styner et al. 2007; Zheng, Dong et al. 2007), where landmark points have to be manually determined based on some specific features of the structures of interest, the proposed method automatically reconstructs landmark points and automatically captures the salient features of surfaces.

As each point on the edge contours is generated by a ray from the relevant X-ray source, the epipolar plane contains the rays linking the $\mathrm{X}$-ray sources to the epipolar tangents points on the image planes. The landmark point is therefore the intersection of a pair of rays from the two distinct X-ray sources. For example, consider a bone-shaped object in Figure 1. Landmark point $\mathbf{f}_{1}$, and the corresponding epipolar tangent points $\mathbf{t}_{1}$ and $\mathbf{t}_{2}$ can be found from the known epipoles, derived from the intersections between the epipolar baseline and the image planes, and edge contours $c_{1}, c_{2}$. Two rays, $\mathbf{r}_{1}, \mathbf{r}_{2}$ from the X-ray sources are defined by linking $\mathbf{X}_{1} \mathbf{t}_{1}$ and $\mathbf{X}_{2} \mathbf{t}_{2}$ respectively. Then landmark point $\mathbf{f}_{1}$ is the intersection of $\mathbf{r}_{1}$ and $\mathbf{r}_{2}$. Note that in practice, it is unlikely that $\mathbf{r}_{1}$ and $\mathbf{r}_{2}$ will intersect exactly. This problem is solved by finding the shortest segment between the two rays and choosing the middle point on the line as the frontier point.

While the plane rotates about the epipolar baseline and touches other parts of the surface, additional landmark points, e.g. $\mathbf{f}_{1}, \mathbf{f}_{2}, \mathbf{f}_{3}$, can be determined. The more complex the surface is, the more additional information is contained in the associated edge contours and therefore the more additional local epipolar tangent points are generated, thus providing the 3D locations of furthur landmark points.

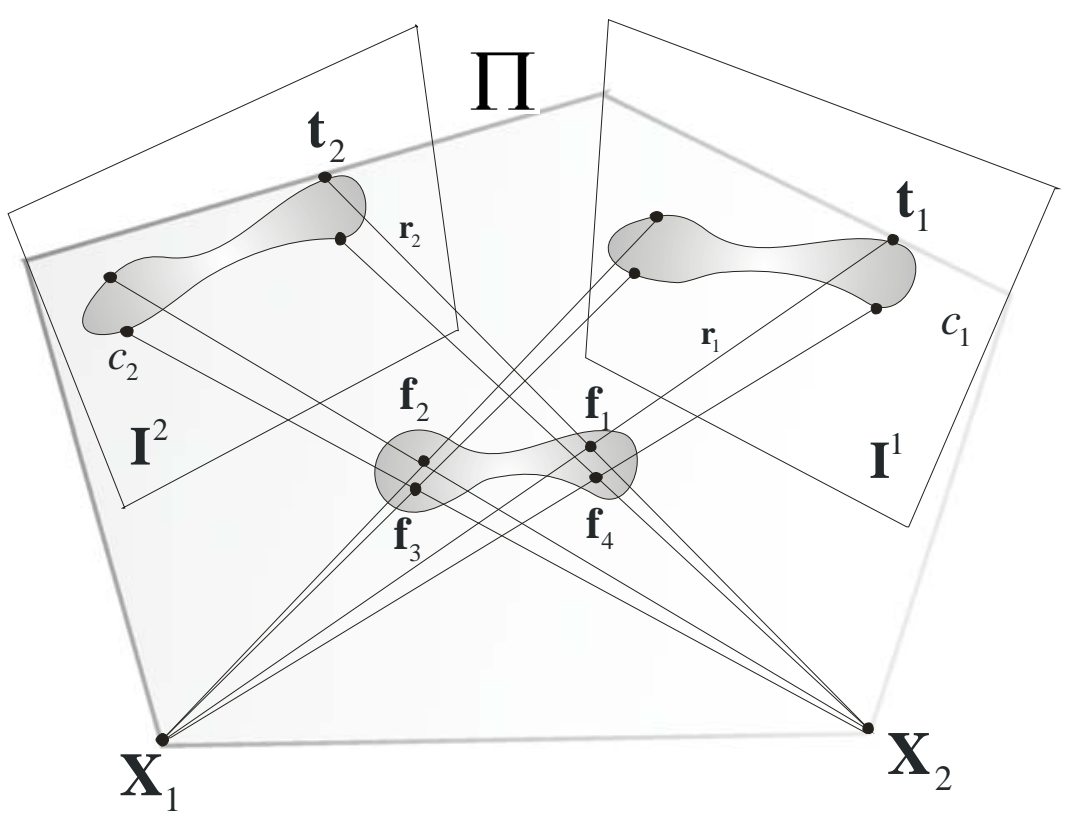


Figure 1. Epipolar plane $\Pi$ rotating about epipolar baseline creating epipolar tangent lines, epipolar tangent points and landmark points.

So far, we have discussed pairing of two X-ray images to reconstructed 3D landmark points. The same fundamentals can further be applied to a set of more than two image. Consider a set of $N_{p l} \mathrm{X}$ ray sources, irradiating surface $S$ from distinct directions, where no potential epipolar baseline passes through $S$. From this set, $N_{p l}\left(N_{p l}-1\right) / 2$ image pairs can be created. If a convex object is projected on to the image planes, then $N_{p l}\left(N_{p l}-1\right)$ landmark points will be determined. If the viewing angles are uniformly distributed in solid angle, smooth shapes, with monotonic change of gradient, result in epipolar tangent points at approximately equal increments of tangent angle on the corresponding edge contour. If $N_{p l}=10$, on each 2D edge contour, interaction with nine other views will establish 18 epipolar tangent points and 18 landmark points, each centered on an arc of $\pm 10^{\circ}$, for a total of $360^{\circ}$. A nonconvex object will generate more epipolar tangent points and landmark points from the pairing.

Figure 2 shows an example, when $N_{p l}=3$ and the 2D X-ray images are $\mathbf{I}^{1}, \mathbf{I}^{2}$ and $\mathbf{I}^{3}$. Three image pairs $\left(\mathbf{I}^{1}, \mathbf{I}^{2}\right),\left(\mathbf{I}^{1}, \mathbf{I}^{3}\right)$ and $\left(\mathbf{I}^{2}, \mathbf{I}^{3}\right)$ can be created. For simplicity, we assume that all the image planes are at right angle to one another. The $\mathrm{X}$-ray sources are at $\infty$ and all $\mathrm{X}$-rays are parallel to the associated image plane's normal. If the object is a sphere, the corresponding edge contours $C_{1}, C_{2}$ and $c_{3}$ are all circles, Fig. 2(a). Six landmark points $\mathbf{f}_{i}, i=1,2,3, \ldots, 6$, can then be determined by the intersections of the relevant rays in the three image pairs as illustrated in Fig. 2(b). Image pairs $\left(\mathbf{I}^{1}, \mathbf{I}^{2}\right.$ ), $\left(\mathbf{I}^{1}, \mathbf{I}^{3}\right)$ and $\left(\mathbf{I}^{2}, \mathbf{I}^{3}\right)$ generate landmark point pairs $\left(\mathbf{f}_{1}, \mathbf{f}_{2}\right),\left(\mathbf{f}_{3}, \mathbf{f}_{4}\right)$ and $\left(\mathbf{f}_{5}, \mathbf{f}_{6}\right)$ respectively. The related epipolar tangent lines and the epipolar tangents points $\mathbf{t}_{j}, j=1,2,3, \ldots, 12$, on the image planes are shown in Fig. 2(c).

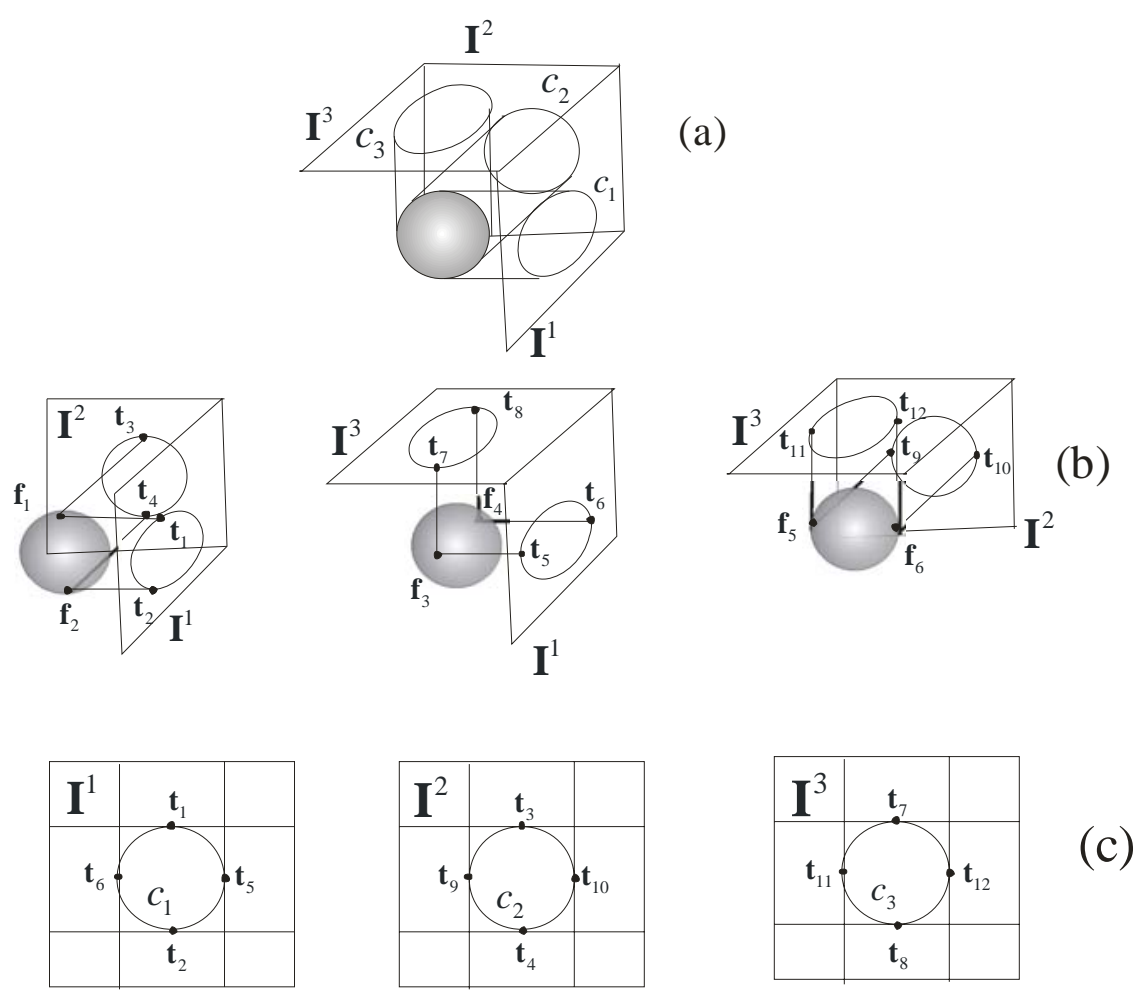

Figure 2. A simple three X-ray source system (a) a sphere is projected onto three image planes $\mathbf{I}^{1}, \mathbf{I}^{2}$ and $\mathbf{I}^{3}$ containing edge contours $C_{1}, C_{2}$ and $C_{3}$ (b) frontier points $\mathbf{f}_{i}, i=1,2,3, \ldots, 6$, are reconstructed from the 
corresponding edge contours (c) distribution of epipolar tangent points $\mathbf{t}_{j}, j=1,2,3, \ldots, 12$, on the corresponding edge contours on the image planes.

\subsection{Data acquisition system geometry design}

In (Prakoonwit and Benjamin 2007), Benjamin and the author presented a full surface reconstruction from a set of 2D conventional X-ray images taken from 10 to 15 projection directions. However, the number of projections is too high for intraoperative applications. Therefore, we propose to use only about 5 projections. This would create a set of surface points, but the information acquired from this limited set of images is not adequate to fully reconstruct a surface model using the method described in (Prakoonwit and Benjamin 2007). Instead, the reconstructed surface points will be used as a set of optimal landmark points which will be extrapolated to create a full surface model using a statistical model as prior knowledge.

\subsubsection{Designing of X-ray projection system}

To make the method feasible for intraoperative applications, we propose the X-ray scanning geometry shown in Figure 3. A single 2D X-ray array detector is located underneath the operating table covering the area of interest. The X-ray base can be installed on the ceiling of the operating theatre, or mounted on a mobile arm. It contains $5 \mathrm{X}$-ray sources with different projection directions as required. The X-ray sources are turn on in turn to sequentially create 2D X-ray images from different projection angles, defined by $\alpha$ and $\beta$. In some applications where multiple $\mathrm{X}$-ray sources may not be economical, a single X-ray source can be used with a mechanism to adjust the X-ray source's position and direction to sequentially create the $\mathrm{X}$-ray images from different projection directions.

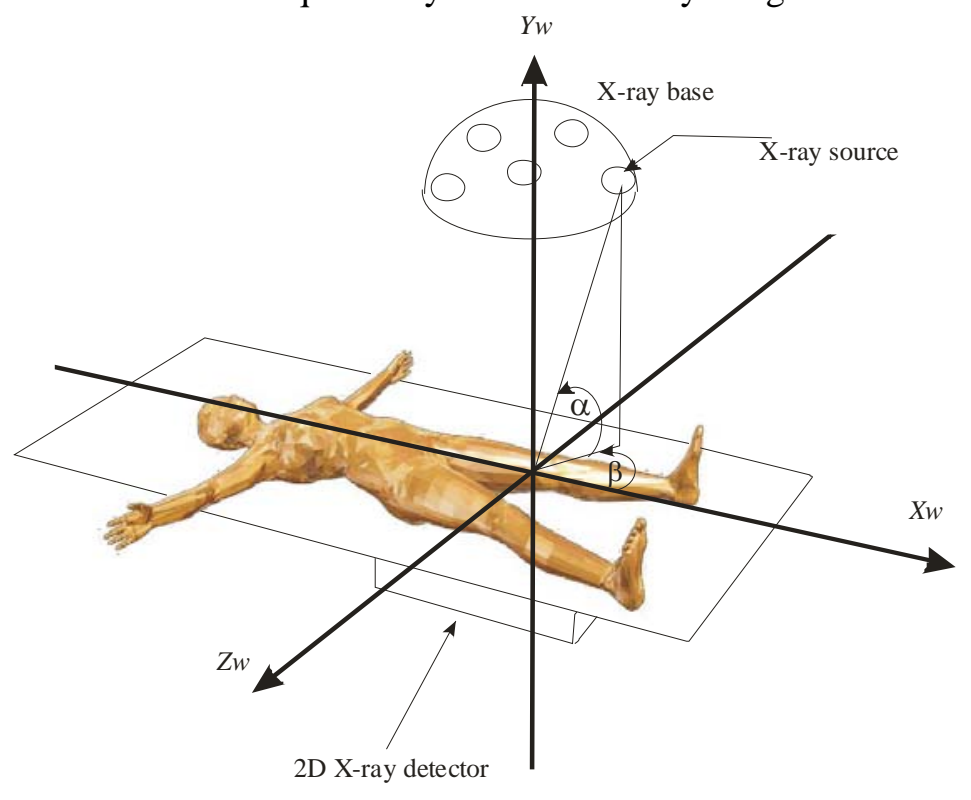

Figure 3. Proposed scanning geometry. An X-ray projection direction is defined by angles $\alpha$ and $\beta$ in $X w, Y w, Z w$ coordinates system.

\subsubsection{X-ray projection angle determination}

In this section, the aim is to create a set of surface points optimally distributed on the object's surface. The optimal distribution means the surface points approximately cluster closely on highly curved 
parts of the surface and are widely spread on smooth or flat parts. To approximately create the optimal distribution of the landmark points, the following condition must be satisfied: $\delta s \propto 1 / \bar{\kappa}$, where $\bar{\kappa}=$ mean curvature and $\delta s$ is a length of an arc on an edge contour $c$ between any two consecutive epipolar tangent points. Thus the length of an arc must be inversely proportional to the local mean curvature. In other words, the higher curvature the shorter distance between two epipolar tangent points. Thus the epipolar tangent points cluster closely in highly curved parts (high $\bar{\kappa}$ ) and widely spread in smooth flat parts (low $\bar{\kappa}$ ) of the edge contour. If the normal vectors at epipolar tangent points are considered instead of the tangents, the gap angle between any two consecutive normal vectors must be a constant. Thus the gap angle $\delta \theta$ between normal vectors $\mathbf{n}_{1}$ and $\mathbf{n}_{2}$ of epipolar tangent points $\mathbf{t}_{1}$ and $\mathbf{t}_{2}$ must be equal to the angle between any other two consecutive normal vectors on a curve segment. Provided that this condition is satisfied, the reconstructed 3D frontier points on the object itself will also approximately gather closely on highly curved parts of the surface and the surface and broadly spread on smooth flat parts.

In $n$-image system, assuming that we select the positions of the X-ray sources optimally, $\delta \theta$, the gap angle for any arc between consecutive epipolar tangent points, on any edge contour in any image plane, must be the same, i.e. $\delta \theta=\pi /\left(N_{p l}-1\right)$, where $N_{p l}$ is the number of X-ray sources.

As the distribution of the reconstructed landmark points depends on the positions of the X-ray image planes and the associated X-ray sources, the problem is how to find a set of X-ray projection directions that create such optimal or near optimal distribution. Especially, when the positions and directions of the X-ray sources are restricted by the geometry of the scanning system such as the one shown in Figure 3.

This problem is similar to the widely studied problems of camera network design and the next best view (Mason and Grun 1995; Mason 1997; Pito 1999; Olague and Mohr 2002). The optimization problem in that case is similar to our problem. The complexity of camera positions is in the class of NP-complete problems (Mason 1997; Olague and Mohr 2002). Many approaches have been developed to find a reasonable optimization method (Mason and Grun 1995; Mason 1997; Olague and Mohr 2002). One needs to search for the set that produces the optimal distribution condition on all image planes. The search space is enormous, non-linear, complex and poorly understood. The problem cannot be easily formulated in terms of mathematical equations in order to use traditional optimization techniques (Olague and Mohr 2002).

Olague and Mohr (Olague and Mohr 2002) successfully used the multi-cellular genetic algorithm, a specialized variant of the standard genetic algorithm. It follows the tree-based genetic programming representation (Koza 1992; Kinner 1994), which is assumed to be known and fixed. In this method, each camera variable is partitioned into a cell. The evolutionary process is applied to each camera separately allowing the appearance of subpopulations with good quality individuals. Binary representation is used in order to allow the algorithm to randomly search the whole space. The multicellular genetic algorithm evaluates the fitness of the whole system of multiple cameras, rather than the fitness of each cell individually. Since the camera placement problem and our X-ray source placement problem are similar, we adopt the same technique. 
The objective is to minimise the error function for the multi-cellular genetic algorithm is defined by

$$
\varepsilon=\left.\overline{|\Delta \omega|}\right|_{\delta \theta}=\frac{1}{N} \sum_{i=1}^{N}\left|\omega_{i}-\delta \theta\right|
$$

where $\omega_{i}$ is the angle between the normal vectors at the two ends of the $i^{\text {th }}$ arc between two consecutive epipolar tangent points, and $N$ is a total number of the arcs on all edge contours on all image planes in the set. In ideal case, $\varepsilon$ is equal to zero. The value of $\omega_{i}$ depends on the projection angles $\alpha$ and $\beta$. It can be calculated using the method explained in the previous section. The ranges of $\alpha$ and $\beta$ are $a \leq \alpha \leq 90^{\circ}, 0^{\circ} \leq \beta \leq 360^{\circ}$ where $a$ is a constant depending on the shape and size of the X-ray scanning geometry. Assuming that the projection distance is defined by the fix geometry of the scanning system and the corresponding projection angles, the parameters to be encoded in the multi-cellular algorithm are $\alpha$ and $\beta$. The detailed method on how to implement the multi-cellular algorithm can be found in (Olague and Mohr 2002).

\subsection{Surface reconstruction}

From the previous section, we now have a set of landmark points distributed on the surface. The next stage is to extrapolate those points to a complete 3D surface model of a bone. The problem statement is shown in Figure 4. However, reconstructing an acceptable 3D surface model from such sparse data is a challenging task as reviewed in Section 1. Normally, without prior knowledge, it is rather impossible or it is not computationally viable to accurately reconstruct a full surface description for our applications. Therefore, a statistically based shape model is considered to be used as prior knowledge. Based on such model, the reconstructed landmark points can be applied to infer the full anatomical information in a robust way.

There are many approaches for constructing statistical shape models. Staib and Duncan (Staib and Duncan 1992) and Szekelely, et al (Szelely, Lelemen et al. 1996) applied Fourier representations for statistical models. Modal analysis can also be applied to create a decomposition of shapes into a basis of fundamental deformations, e.g. (Pentland and Scalroff 1991). It is also feasible to use surface features such as crest-lines and to perform modal analysis on the features (Subsol, Thirion et al. 1996). Another approach is to consider a statistical model with modal representation based on Principal Component Analysis (PCA). This method was first proposed by Cootes, et al (Cootes, Taylor et al. 1995). Since then, it has been widely used in model reconstruction and object recognition. The method has been proven to be suitable for many orthopaedic applications, e.g. (Fleute, Lavallee et al. 1999; Rajamani, Styner et al. 2007; Zheng, Dong et al. 2007). This article is, therefore, based on the statistical shape model approach (Cootes, Taylor et al. 1995; Zheng, Dong et al. 2007). 

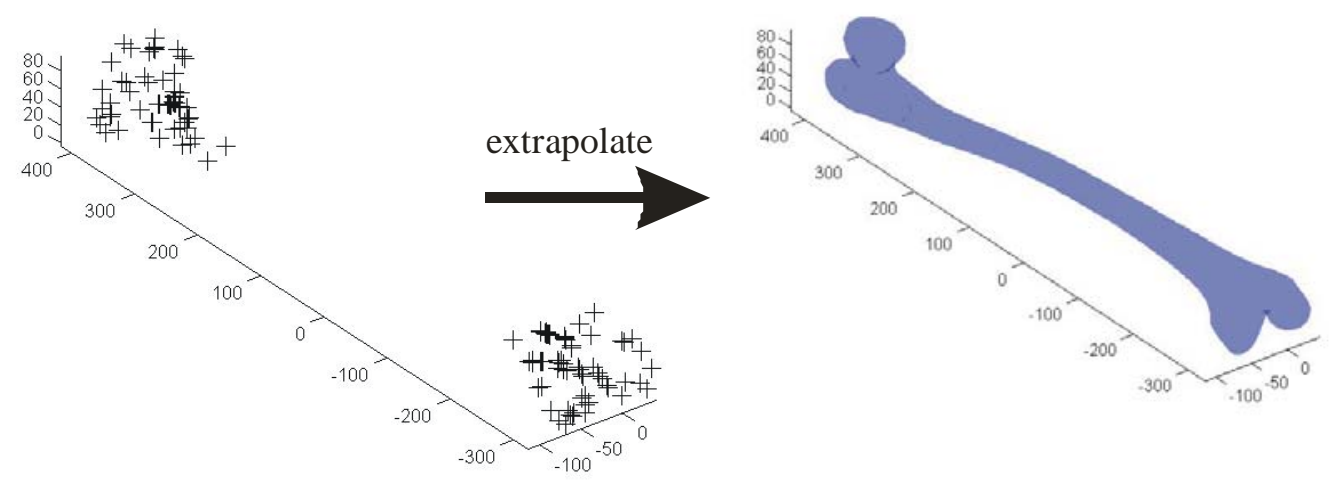

Figure 4. Problem: how to extrapolate sparse surface points to a complete bone surface?

\subsubsection{Dense-point distribution model construction}

In this work, we use a dense-point distribution model (DPDM) (Zheng, Dong et al. 2007), which is a type of statistical shape models, in our surface reconstruction. Building the statistical dense-point distribution model requires the following steps: acquiring, aligning, matching the training shapes; and Dense-Point Distribution model construction using PCA. The two steps are detailed as follows.

(i) Acquiring, aligning and matching the training shapes. A set of 30 computer-generated surface models of femurs and knee joint bones with randomly varied shapes and sizes are used as the training shapes for building the statistical shape model representing femurs and knee joint bones. Each individual computer-generated surface model is represented by a set of triangle mesh containing 4484 triangular facets and 2244 vertices. The shapes of the bones are randomly deformed to represent the possible variations in the real population. Some of the femurs models are shown in Figure 5.

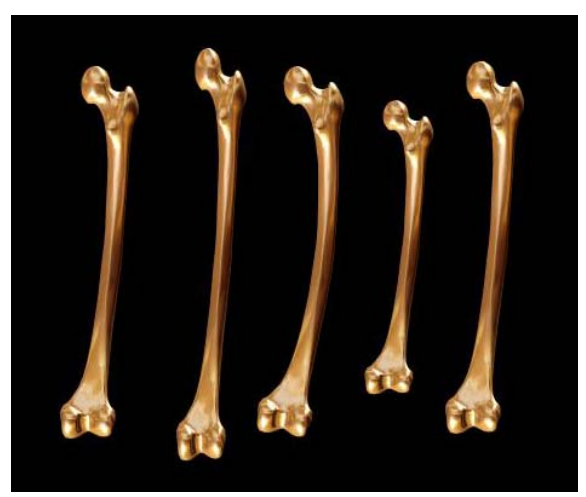

Figure 5. Some computer generated femurs in the training set.

Let $\mathbf{v r} \mathbf{r}_{i}=\left[x r_{i, 0}, y r_{i, 0}, z r_{i, 0}, x r_{i, 1}, y r_{i, 1}, z r_{i, 1}, \ldots, x r_{i, N r-1}, y r_{i, N r-1}, z r_{i, N r-1}\right]^{T}, i=0,1, \ldots, M-1$, be a vector representing $N r$ vertices of the $i^{\text {th }}$ surface model in the set of $M$ training models where, in this case, $M$ $=30$ and $N r=2244$. The 3D position of $n^{\text {th }}$ vertex of surface model $i^{\text {th }}$ is defined by $\left[x r_{i, n}, y r_{i, n}, z r_{i, n}\right]$.

However, DPDM requires more vertices to create a dense cloud of vertices on the surface. This can be obtained by iteratively refining the training computer-generated surface models to create the DPDM. The refining method proposed in (Zheng, Dong et al. 2007) is adopted in this work. It uses a simple 
subdivision scheme call loop scheme, (Loop 1987), to guarantee that the surface mesh is smooth. The level of subdivision and the number of dense vertices required depend on the application. Zhen, et al, (Zheng, Dong et al. 2007) recommends that, for this type of applications, the maximum edge length of all triangles should be less than $1.5 \mathrm{~mm}$. Therefore, approximately one-level or two-level subdivision is found to be adequate.

Having been subdivided, each surface model is now represented by a set of dense vertices $\mathbf{v}_{i}^{\prime}=\left[x_{i, 0}, y_{i, 0}, z_{i, 0}, x_{i, 0}, y_{i, 0}, z_{i, 0}, \ldots, x_{i, N-1}, y_{i, N-1}, z_{i, N-1}\right]^{T}$ where $i=0,1, \ldots, M-1$ and $N=$ number of vertices after the subdivision process, $N \gg N_{r}$.

The next stage is to align all the femur surfaces in the training set. The alignment method used in (Szelely, Lelemen et al. 1996; Fleute, Lavallee et al. 1999) is implemented in this work. The method performs least-squares minimisation of the distances between a sparse and unorganised set of vertices and a dense set of vertices. Therefore, only the template model is required to be dense-point model, the rest can be the original models. This would reduce the computational workload in the alignment process. Therefore, suppose surface $\mathbf{v}_{0}^{\prime}$ is selected to be a template, then surfaces $\mathbf{v r}_{j}, j=1,2, \ldots, M-1$ can be aligned with $\mathbf{v}_{0}^{\prime}$, one by one. The alignment parameters, can then be used to align all the dense-point shapes $\mathbf{v}_{i}^{\prime}, i=1,2, \ldots, M-1$, creating a new set of training 3D shapes represented by $\mathbf{v}_{i}, i=$ $0,1,2, \ldots, M-1$ where $\mathbf{v}_{0}=\mathbf{v}_{0}^{\prime}$. The details of the alignment algorithm can be found in (Fleute, Lavallee et al. 1999).

(ii) Dense-point distribution model construction using PCA. Once all the surface vertices of the models in the training set have been aligned, the statistics of the set of aligned 3D models can be captured by a dense-point distribution model. The DPDM is created by applying PCA on aligned $\mathbf{v}_{i}$ as described in (Cootes, Taylor et al. 1995; Fleute, Lavallee et al. 1999; Zheng, Dong et al. 2007). The covariance matrix, $\mathbf{S}$, can be calculated using

$$
\mathbf{S}=\frac{1}{(M-1)} \sum_{i=0}^{M-1} d \mathbf{v}_{i} \cdot d \mathbf{v}_{i}^{T}
$$

where $d \mathbf{v}_{i}=\mathbf{v}_{i}-\overline{\mathbf{v}}$ and $\overline{\mathbf{v}}=\frac{1}{M} \sum_{i=0}^{(M-1)} \mathbf{v}_{i}$. Following the method in (Zheng, Dong et al. 2007), the principle axes are described by the unit eigenvectors, $\mathbf{p}_{i}$, of $\mathbf{S}$ such that

$$
\mathbf{S} \cdot \mathbf{p}_{i}=\lambda_{i} \cdot \mathbf{p}_{i}, i=0, \ldots, M^{\prime}-1
$$

where $\lambda_{i}$ is the $i^{\text {th }}$ eigenvectors of $\mathbf{S}, \lambda_{i} \geq \lambda_{i+1}$ and $M^{\prime} \leq M-1$.

A 3D model in the training set can be approximated using the mean model and a linear combinations with weights $\mathbf{w}=\left[w_{0}, w_{1}, \ldots, w_{m^{\prime}-1}\right]^{T},-3 \sqrt{\lambda_{i}} \leq w_{i} \leq 3 \sqrt{\lambda_{i}}$ (Cootes, Taylor et al. 1995), obtained from the first $M^{\prime}$ modes:

$$
\mathbf{v}=\overline{\mathbf{v}}+\sum_{i=0}^{M^{\prime}-1}\left(w_{i} \cdot \mathbf{p}_{i}\right)
$$




\subsubsection{Model fitting}

Given reconstructed landmark points $\mathbf{f}_{j}=\left[\begin{array}{lll}x_{j} & y_{j} & z_{j}\end{array}\right]^{T}, j=0,1, \ldots, N f-1$ and $N f \ll N$, the whole surface can be reconstructed by fitting the DPDM represented by equation (4) to the landmark points $\mathbf{f}$. However, before model fitting can be done, the affine registration has to be employed to align the reconstructed frontier points to the mean surface model of the DPDM.

This kind of registration is a well-known problem. There are many different methods available. In this work, the ICP algorithm (Besl and McKay 1992; Chen and Medioni 1992; Zheng, Dong et al. 2006; Zheng, Dong et al. 2007), which is one of the most widely used methods, is implemented. The ICP algorithm relies on the search of pairs of closest vertices, and the computation of a paired-point matching transformation. The resulting transformation is then applied to one set of points. The process is progress iteratively until convergence. To prevent the ICP algorithm from converging to a local minimum, we use a set of landmark points on the mean surface model and the reconstructed landmark points, to initialise the registration procedure. The aligned reconstructed frontier points are represented by $\mathbf{f}_{j}^{\prime}=\left[\begin{array}{lll}x_{j} & y_{j} & z_{j}\end{array}\right]^{T}, j=0,1, \ldots, N f-1$.

After the reconstructed frontier points have been aligned with the mean surface model, the homologous points of the aligned reconstructed landmark points on the dense mean surface model of the DPDM can be determined. Hence the DPDM can be use to represent the reconstructed surface by minimising the following error function (Rajamani, Styner et al. 2004; Zheng, Dong et al. 2007).

$$
E_{w}=\rho \cdot \sum_{j=0}^{N f-1}\left\{\left\|\mathbf{f}_{j}^{\prime}-\left[\left(\overline{\mathbf{v}}_{k}\right)_{j}+\sum_{i=0}^{M^{\prime}-1}\left(w_{i} \cdot \mathbf{p}_{i}(k)\right)\right]\right\|^{2}\right\}+\sum_{i=0}^{M^{\prime}-1}\left(\frac{w_{i}^{2}}{\lambda_{i}}\right)
$$

where $\rho=$ a factor that controls the relative weighting between the two terms, $\left(\overline{\mathbf{v}}_{k}\right)_{j}$ represents the $k^{\text {th }}$ point $\overline{\mathbf{v}}_{k}$ on the mean surface model $\overline{\mathbf{v}}$ of the DPDM is the closest point to the $j^{\text {th }}$ aligned reconstructed frontier point $\mathbf{f}_{j}^{\prime}$. The details on how to determine $\rho$ and optimise Eq D to estimate the full surface model which fits the reconstructed frontier points can be found in (Zheng, Dong et al. 2007).

\section{Multiple bones: bone and edge contour identification}

So far, we have considered only a single bone in the volume of interest. The object can easily be identified and all the edges can be assumed to be part of the object. In practical circumstances, there are many bones and other objects in the volume of interest. The situation is more complicated. This is not a trivial task. Objects in X-ray images are overlapped and superimposed. To automatically extract and reconstruct only the object of interest, an effective and robust object identification method has to be developed to identify only the object's edges among others in the images. In our earlier work (Prakoonwit and Benjamin 2007), an algorithm was proposed. However, the method was developed for general object reconstruction without object prior knowledge and the computational cost to implement the method is high. This make it unsuitable for intraoperative applications.

In this case, prior knowledge about each bone to be reconstructed is known. A new method to identify each bone's edge contours can be developed using this prior knowledge. Each computer generated 3D 
bone training model described in Section 3.4.1 can be used to identify appropriate edge contours in 2D images.

In this paper, a new method for bone edge contour identification is presented. The edge contours on a 2D X-ray image from a 3D bone training model should be approximately similar to those edge contours from a real bone of the same type with approximately the same position and direction. The information can then be used to identify edge contours from different objects in the volume of interest. For example, in Figure 6, there are edge contours (solid lines) from a femur and a patella. To identify that contour $\mathrm{C} 2$ belongs to the femur, not the patella, the edge contours C1 (dotted line) from a computer generated 3D femur model can be created on the 2D image. Contour $\mathrm{C} 1$ is more similar to C2 than C3, therefore, C2 can be identified as the femur contour. Patella contour C3 can be identified in the same manner.

To measure the similarity between two edged contours, the distance between the contours can be used (Bingham and Li 2006; Zhu and Li 2011). Note that to be able to measure the similarity, it is necessary to find the approximate 3D rigid transformation (rotation $\mathbf{R}$ and translation $\mathbf{T}$ ), (Markelj, Tomazvic et al. 2012), so that the 3D model can be approximately aligned with the real bone which generates the edge contours to be identified. This is done simultaneously with the contour identification process. The objective function to be minimised can be established as follows:

$$
\min E=\min \left(\left|C_{m}-C_{c n}\right|\right)
$$

where $\left|C_{m}-C_{c n}\right|$ is the distance between edge contours $C_{m}$ and $C_{c n}, C_{m}$ is the edge contour of the 3D training model and $C_{c n}, c n=1,2,3 \ldots$ are contours from real bones on the image. As described earlier, a 3D computer generated training surface model is represented by a set of triangular mesh. $C_{m}$ can, therefore, be approximately determined by projecting the edges shared by two triangles with normal pointing toward and the other pointing away from the X-ray source. The 3D rigid transformation ( $\mathbf{R}$ and $\mathbf{T}$ ) is also found simultaneously in the minimisation process with $\mathbf{v r ^ { \prime }}=\mathbf{R} \cdot \mathbf{v r}+\mathbf{T}$, where $\mathbf{v r}$ is the model's set of vertices as described in Section 3.4.1.

In this work, there are $N_{p l} 2 \mathrm{D}$ X-ray images used. To integrate all information from all images, the objective function is then formulated as:

$$
\min E=\sum_{p l=1}^{N p l} \min \left(\left|C_{m}^{p l}-C_{c n}^{p l}\right|\right)
$$

where $N p l$ is the number of views or $2 \mathrm{D}$ X-ray images used and $p l$ represents $p l$ th $2 \mathrm{D}$ image. $C_{c n}$ which produces min $E$ can be identified as the edge contours of the same type of bone as the model which generates $C_{m}$ and can be used to reconstruct the corresponding full 3D surface model. 


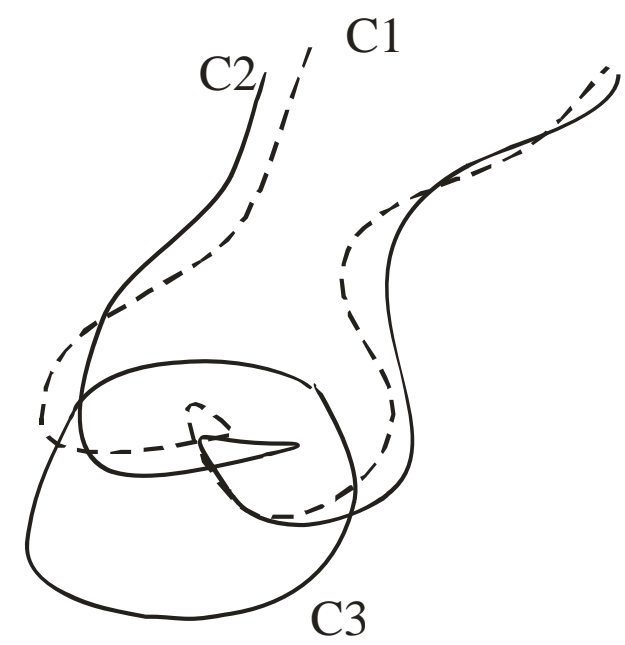

Figure 6. Edge contours from multiple objects (Femur and Patella) can be identified by using the prior knowledge about the shape of the object. Edge contour C1 from a computer generated 3D model can be used to identify C2 as a Femur contour, not a Patella contour.

\subsection{Distance between edge contours}

To measure the distance between two edge contours, a large number of points along the contours are selected (Zhu and Li 2011; Baka, Bruijne et al. 2012) to calculate the distance. However, this is a computationally expensive step in the minimisation process. As the main aim is identifying edge contours, to decrease computational load, the number of points used is reduced by employing appropriately distributed clusters of smaller numbers of points along the edge contours. To include significant features of the edge contours in the process, the points should gather closely on highly curved parts and broadly smooth spread on smooth flat parts. This is the same condition described in Section 3.3.2 where $\delta s \propto 1 / \bar{\kappa}$, where $\bar{\kappa}=$ mean curvature and $\delta s$ is a length of an arc on an edge contour $C$ between any two consecutive points. Thus the gap angle $\delta \theta$ between the two normal vectors of two any consecutive points on the contour must be constant. The number of points can be adjusted by varying $\delta \theta$. The similarity between the two edge contours can then be approximated by measuring the distances between the two set of point clusters.

\subsection{Complex cases}

In most cases, the proposed use of the point clusters is sufficient to identify edge contours. However, there may be a situation where edge contours are complex or heavily super imposed. The clustering method alone may not produce satisfactory results or the value $E$ of the objective function is not sufficiently low. $\delta \theta$ can be decreased to increase the number of matching points. If this still does not suffice, the method suggested in (Baka, Bruijne et al. 2012) can be modified and adopted as an extra process.

In this case, the corresponding vertices in the training 3D model set used in the statistical shape building are employed to calculate matching scores. Instead of the function in Eq 7, The objective function to be minimised can then be defined as follows (Baka, Bruijne et al. 2012):

$$
\min E=\min \left(\frac{1}{N_{p l} \cdot N_{c v}} \sum_{i=1}^{N_{p l}} \sum_{j=1}^{N_{c v}} \Psi\left(p_{j}\right)\right),
$$


where $N_{p l}$ is the number of 2D X-ray, and $N_{c v}$ is the number of corresponding vertices $p_{j}$. $\Psi$ is the matching score defined as

$$
\Psi\left(p_{j}\right)=1-o_{i}\left(p_{j}\right) * w_{i}\left(p_{j}\right) * \operatorname{Dist}_{i}\left(p_{j}\right)
$$

where $w=\left\{\begin{array}{c}0, \text { if } \alpha>90^{\circ} \\ \cos (\alpha), \text { otherwise }\end{array}, w_{i}\left(p_{j}\right) \in[0,1]\right.$ and

$\operatorname{Dist}_{i}\left(p_{j}\right)=\exp \left(-\frac{d_{i}\left(p_{j}\right)}{s}\right)$

$o_{i}\left(p_{j}\right)$ marks the points on the occluding contours on the 3D surface model such that it results in 1 if the projections of the points are within the field of view of projection $i$, otherwise it will be zero. The points on the occluding contours are the points where X-rays are tangential to the surface. On the model, the occluding points can be approximated by the points on the triangular facets' edges which are shared by two facets with on normal vector pointing toward and the other pointing away from the $\mathrm{X}$-ray source. $d_{i}\left(p_{j}\right)$ is the distance in $3 \mathrm{D}$ from vertex $p_{j}$ to the ray connecting the nearest point on the corresponding edge contour of the projected $p_{j}$ with the X-ray source. Parameter $v$ controls the smoothness of the function and $\alpha$ denotes the angle between the gradient of the $\mathrm{X}$-ray image at the edge contour location and the projected normal vector of the 3D model surface at $p_{j}$. More details can be found in (Baka, Bruijne et al. 2012).

\section{Experimental results and discussion}

Computational experiments have been performed to demonstrate the capability of the proposed method. Femur bones and a set of knee joint bones have been selected as test objects. The ranges of projection angle used are $50^{\circ} \leq \alpha \leq 90^{\circ}$ and $0^{\circ} \leq \beta \leq 360^{\circ}$.

\subsection{Single bone}

A set of 30 computer generated femurs are used as a training set as described earlier. A computer generated test femur to be reconstructed is shown in Figure 7 (a). Note that, the test femur is different from those in the training set.

2D X-ray images of the test femur taken at 5 different projection angles determined by the method described in section 3.3.2 are simulated. The edge contours of the femur on all images are determined by the method described in Section 3.1. The edge contours are used for the landmark vertex reconstruction as explained in Section 3.2. The reconstructed landmark vertices and the 3D surface reconstruction using the DPDM to fit the landmark vertices can be found in Figure 7(b). It demonstrates that the landmark vertices are approximately well distributed on the surface. The error distance distribution of the reconstructed 3D surface is shown in Figure 8. The error distance is defined as the shortest distance from the all vertices of the reconstructed femur to the original test femur. 

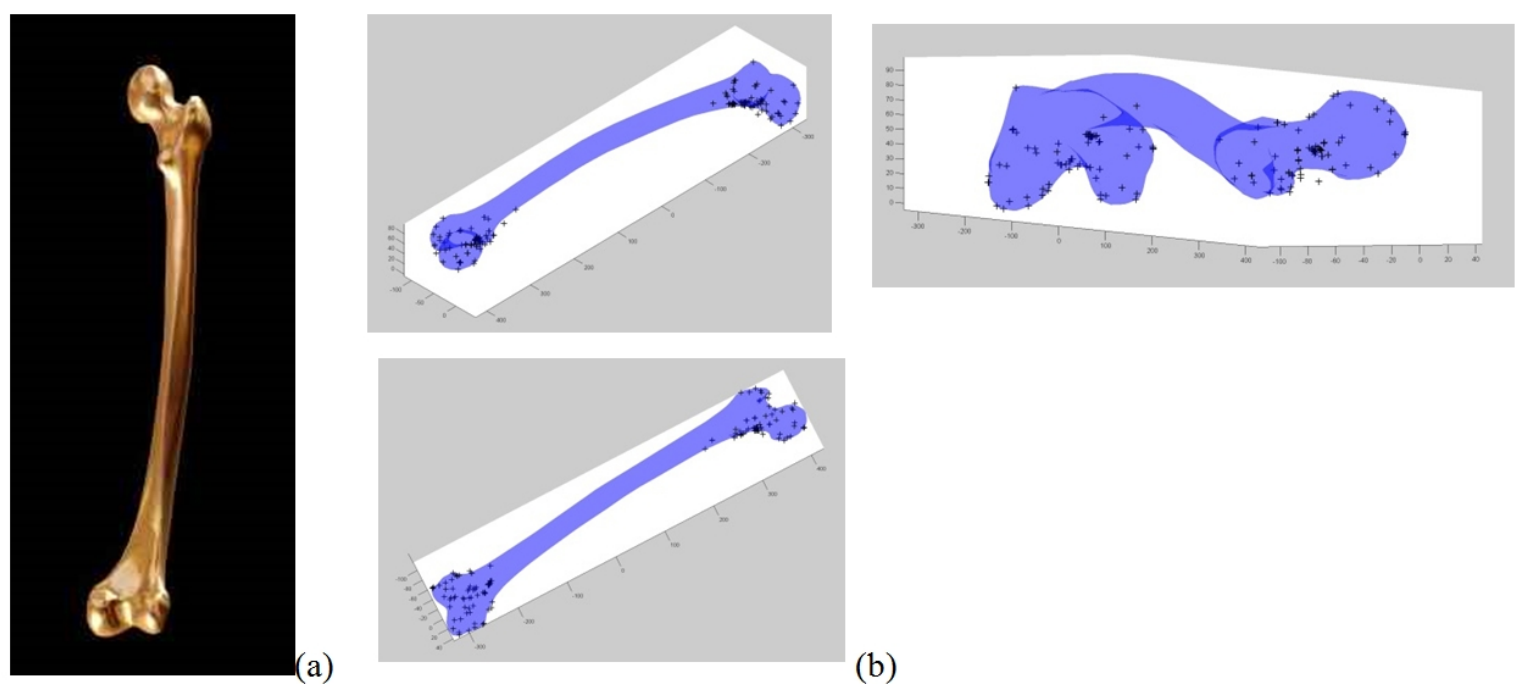

(b)

Figure 7. (a) Test femur to be reconstructed (b) 3D reconstructed surface and the reconstructed landmark vertices viewed from different directions.

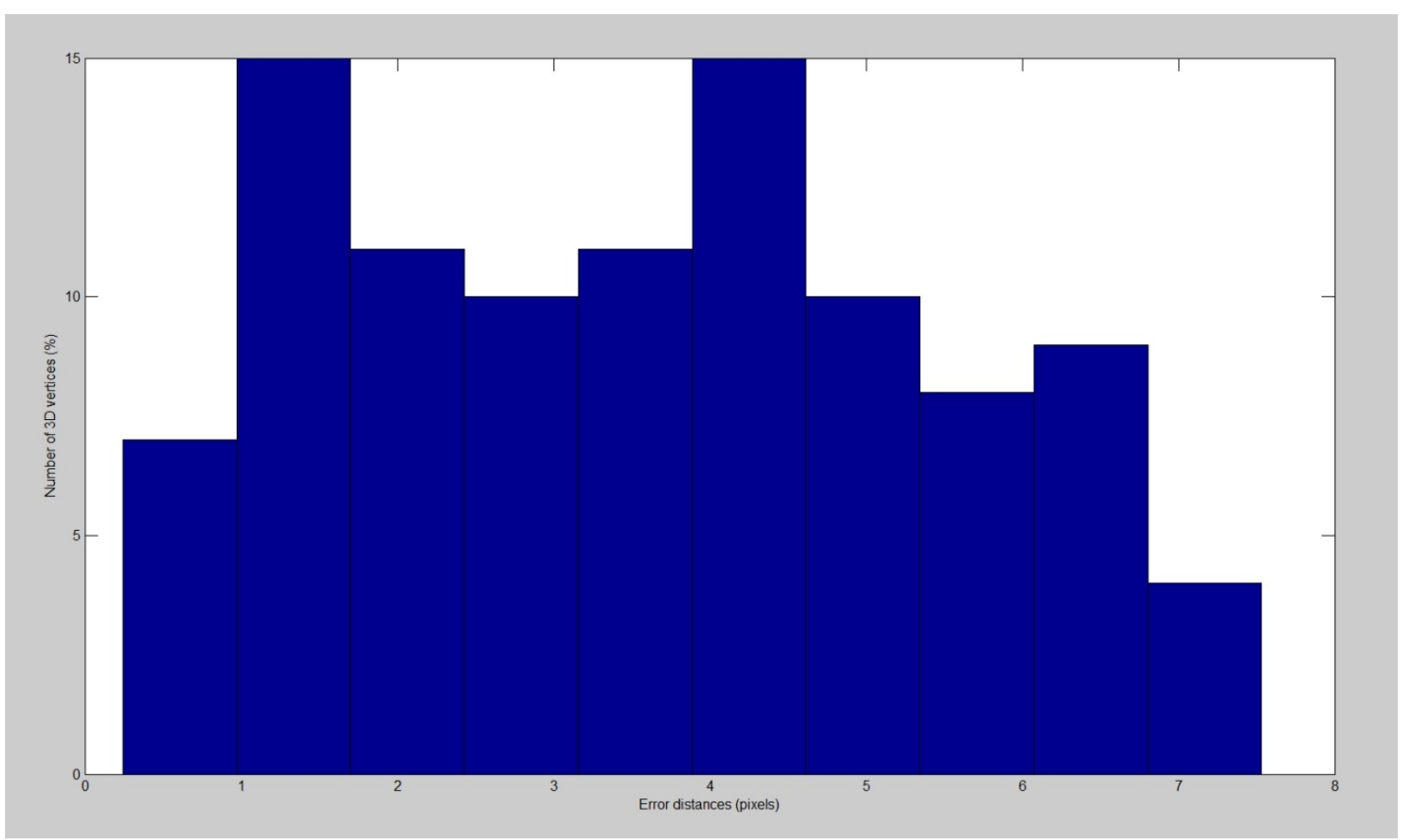

Figure 8. Error distribution of the reconstructed femur compared with the original test femur.

\subsection{Multiple bones}

In case multiple objects, a set of 30 computer generated knee joint consisting of Femurs, Patellas, Tibias and Fibulas are used as a training set. A computer generated test knee joint to be reconstructed, which is different from those in the training set, is shown in Figure 9 (a). As the joint consists of multiple objects, their overlapped and superimposed edge contours in the corresponding X-ray images 
have to be identified by the new method described in Section 4. The resulting edge contours for the Femurs, Tibia, Patella and Fibula successfully identified by the method are shown in Figure 9 (b)-(e) respectively.
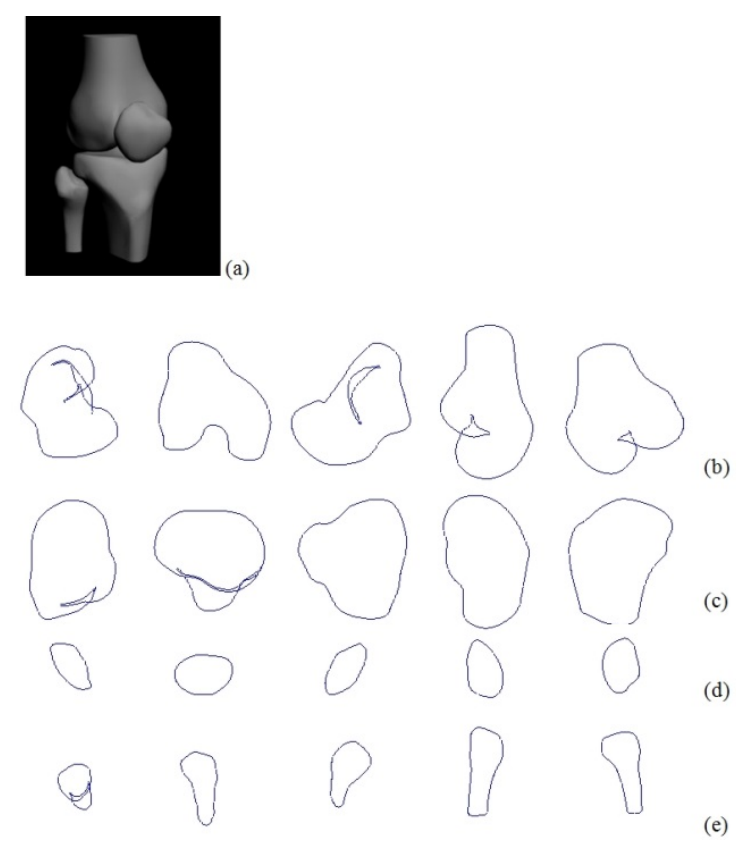

Figure 9. (a) Test knee joint to be reconstructed. (b)-(e) Corresponding edge contours for each bone on the five $2 D X$-ray images.

The 3D surface reconstruction for each bone using the DPDM to fit the landmark vertices can be found in Figure 10. The error distance distribution of all the reconstructed 3D surface is presented in Fig 11.
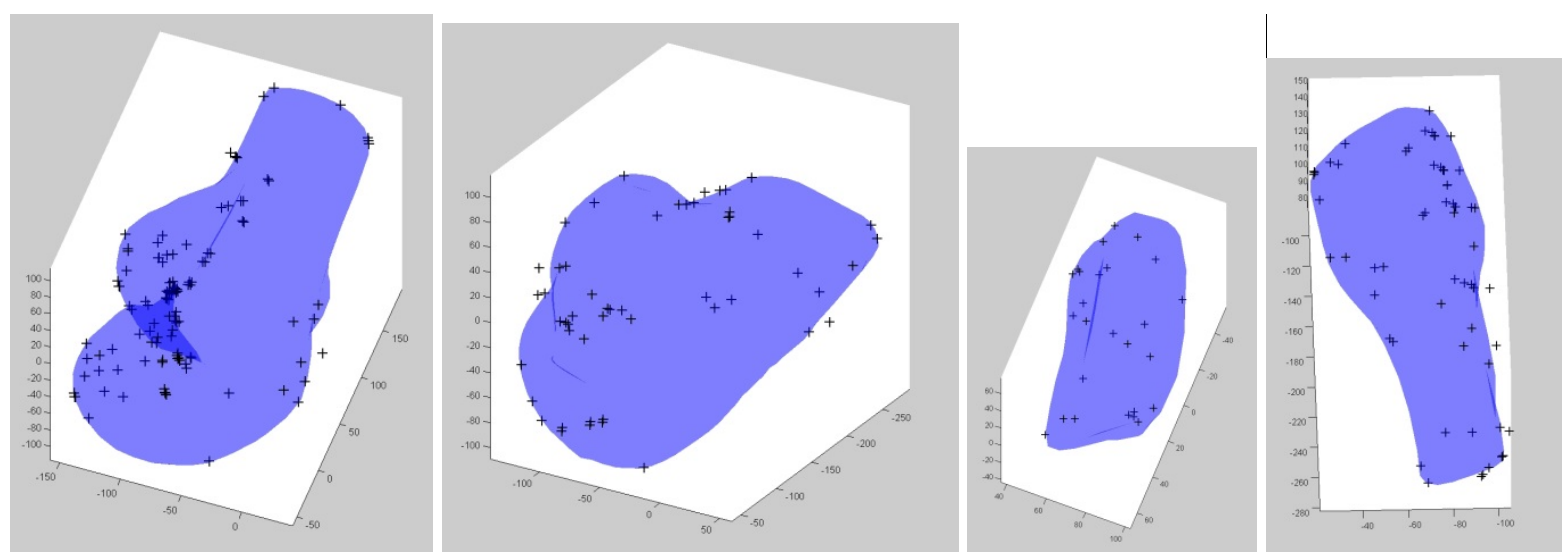

Figure 10. 3D reconstructed surfaces of the Femur, Tibia, Patella, Fibula and the reconstructed landmark vertices. 


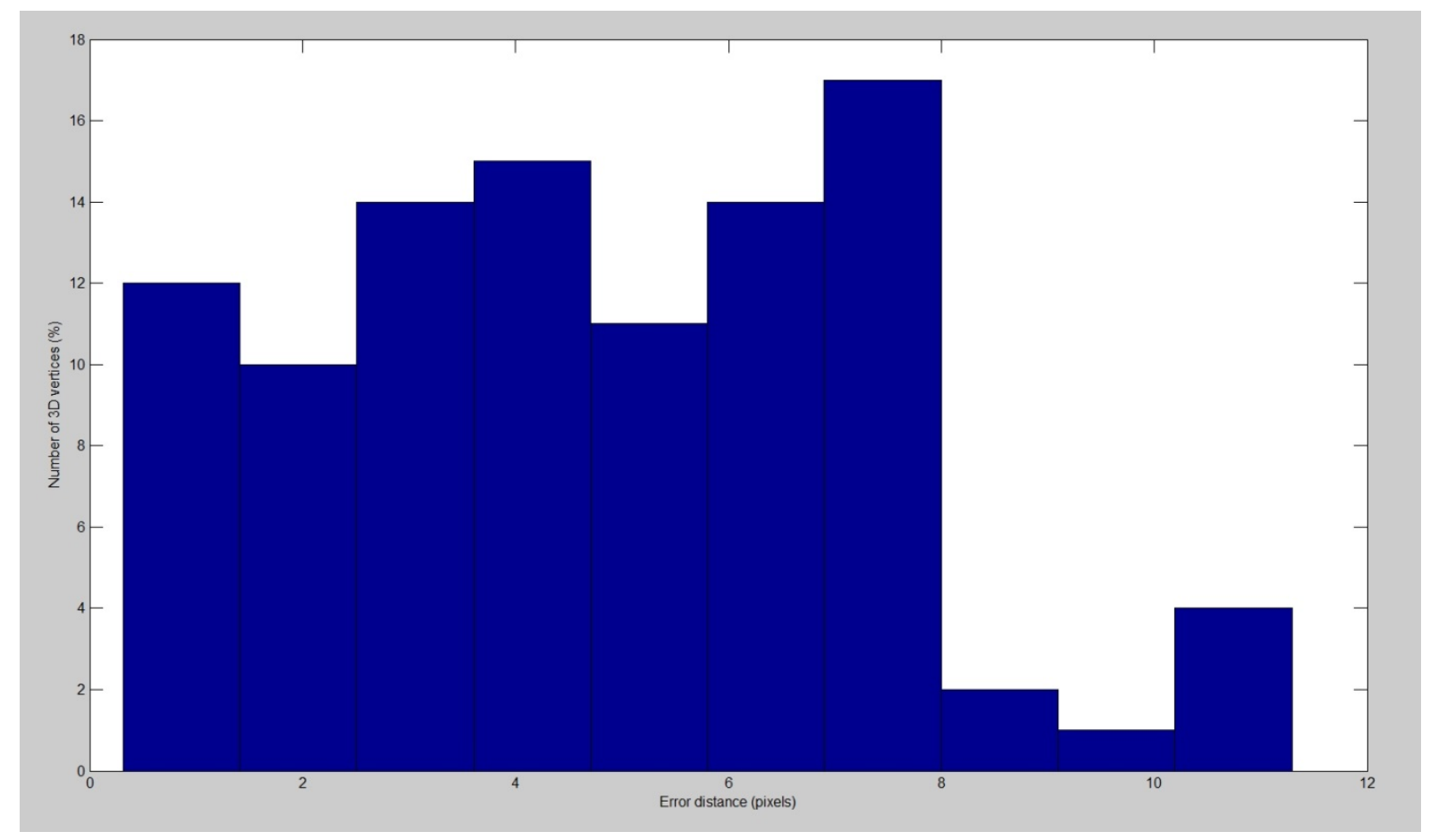

Figure 11. Error distribution of the reconstructed femur compared with the original test femur.

The results show that the proposed method could produce reasonable 3D surfaces of the test objects. The results are more accurate than those reported in our previous work (Prakoonwit 2011). The landmark vertices are automatically generated and adapted to effectively capture the salient features of the bones. In most cases, there are approximately more landmark vertices on the detailed or highly curved parts of the surface and less landmark vertices on the less detailed or flat parts of the surface. This is to guarantee that all the important features of the surface are captured and represented for the model fitting process. However, the distribution of the landmark vertices depends on the ranges of the projection angles $\alpha$ and $\beta$. Ideally, the ranges should be $0 \leq \alpha \leq 90^{\circ}$ and $0 \leq \beta \leq 360^{\circ}$. Due to the scanning geometry, the full range of $\alpha$ would not be feasible for intraoperative applications. The results show that the rage of $50^{\circ} \leq \alpha \leq 90^{\circ}$ is still feasible, even though the distribution of landmark vertices are not completely perfect.

\section{Future research direction}

In this article, a novel approach towards 3D reconstruction using conventional X-ray images for intraoperative orthopaedic applications is presented. The method still has some main limitations which require some future research in the following aspects.

\subsection{Object identification and segmentation}

There is still room for improvement in this part. There are cases where only some parts of objects are visible in X-ray images. The current algorithm proposed in this article may not produce accurate results. This requires further studies. In addition, the method also relies heavily on the edge contours of objects. Therefore, it should work well only on high density objects or tissues such as bones where edges clearly appear on a 2D X-ray image. Poor quality X-ray may not contain all edge contours required by the method proposed.

\subsection{Statistical shape model fitting}


Even though Statistical shape modelling is a robust tool for 3D surface reconstruction from sparse vertices, there is a major limitation. Instances of the model can only be deformed in ways found in the training set. That means the method cannot reconstruct any pathological conditions or deformations which are not included in the training set. Any substantial changes to a bone during an operation may also cause the reconstruction process to fail. To solve this problem, it is suggested that the edges contours from 2D X-ray images can be used to adjust the statistical shape model before fitting it to landmark vertices.

\section{Key Terms}

3D reconstruction, $\mathrm{X}$-ray, intraoperative, statistical model, sparse vertices, surface, epipolar

\section{References}

Abidi, B., M. Mitckes, et al. (2003). Grayscale enhancement techniques of X-ray images for carryon luggage. Proc. of SPIE 6th International Conference on Quality Control by Artificial Vision. Gatlinburg, TN, USA. 5132: 579-591.

Atesok, K., J. Finkelstein, et al. (2007). "The use of intraoperative three-dimensional imaging (ISO-C-3D) in fixation of intraarticular fractures." Injury, International Jouranl of the Care of the Injured 38: 1163-1169.

Baka, N., M. d. Bruijne, et al. (2012). "Statistical shape model-based femur kinematics from biplan fluoroscopy." IEEE Transactions on Medical Imaging 31(8): 1573-1583.

Benameur, S., M. Mignotte, et al. (2005). "A herarchical statistical modeling approach for the unsupervised 3D biplanar reconstruction of the scoliotic spine." IEEE Transactions on Biomedical Engineering 52(12): 2041-2057.

benameur, S., M. Mignotte, et al. (2003). "3D/2D registration and segmentation of scoliotic vertebrae using statistical models." Comput. Med. Imag. Graph 27: 321-337.

Besl, P. and N. D. McKay (1992). "A method for registration of 3D shapes." IEEE Transactions on Pattern Analysis and Machine Intelligence 14(2): 239-256.

Bingham, J. and G. Li (2006). "An optimized image matching method for determining in vivo TKA kinematics with a dual-orthogonal fluoroscopic imaging system." Journal of Biomechanical Engineering 128.

Canny, J. F. (1986). "A computational approach to edge detection." IEEE Transactions on Pattern Analysis and Machine Intelligence 8(6): 112-131.

Chan, C. S., P. J. Edwards, et al. (2003). "Integration of ultrasound based registration with statistical shape models for computer-assisted orthopaedic surgery." Proceedings of SPIE Medical Imaging: 414-424.

Chen, Y. and G. Medioni (1992). "Object modeling by registratoin of multiple range images." Image and Visual Computing 10(3): 145-155.

Cootes, T. F., C. J. Taylor, et al. (1995). "Active shape models - their training and application." Computer Vision and Image Understanding 61(1): 38-59. 
Desolneux, A., L. Moisan, et al. (2008). From Gestalt Theory to Image Analysis: a Probabilistic Approach. New York, Springer Verlag.

Dryden, I. and K. Mardia (1998). Statistical Shape Analysis, John Wiley and Sons.

Fleute, M. and S. Lavallee (1998). Building a complete surface model from sparse data using statistical shape models: application to computer assisted knee surgery system. Proc. 1st Int. Conf. Medical Image Computing and Computer-Assisted Intervention. 1496: 879-887.

Fleute, M. and S. Lavallee (1999). Nonrigid 3D/2D registration of images using statistical models. 2nd International Conference on Medical Image Computing and Computer-Assisted Intervention. 1679: 138-147.

Fleute, M., S. Lavallee, et al. (1999). "Incorporating a statistically based shape model into a system for computer-assisted anterior cruciate ligament surgery." Medical Image Analysis 3(3): 209-222.

Kang, X. (2011). Feature-based 2D-3D registration and 3D reconstruction from a limited number of images via statistical inference for image-guided interventions. Department of Orthopaedics and Traumatology. Hong Kong, The University of Hong Kong. PhD: 179.

Kinner, K. E. (1994). Advances in Genetic Programming. Cambridge, MA, MIT Press.

Kovesi, P. D. (1999). "Image features from phase congruency." Videre J. Comput. Vis. Res. 1(2): $1-26$.

Koza, J. R. (1992). Genetic Programming, On the Programming of Computers by Means of Natural Selection. Cambridge, MA, MIT Press.

Kurazume, R., K. Kakamura, et al. (2009). "3D reconstruction of a femoral shape using a parametric model and two 2D fluoroscopic images." Comput. Vis. Image Understand. 113(2): 202-211.

Lamecker, H., T. H. Wenckeback, et al. (2006). Atlas-based 3D-shape reconstruction from X-ray images. Proc 18th Int. Conf. Pattern Recognition. 1: 371-374.

Loop, C. T. (1987). Smooth subdivision surfaces based on triangles. Mathematics. Salt Lake, University of Utha. Master of Science.

Markelj, P., D. Tomazvic, et al. (2012). "A review of 3D/2D registration methods for imageguided interventions." Medical Image Analysis 16(3): 642-661.

Mason, S. (1997). "Heuristic reasoning strategy for automated sensor placement." Photogramm. Eng. Remote Sensing 63(9): 1093-1102.

Mason, S. and A. Grun (1995). "Automatic sensor placement for accurate dimensional inspection." Computer Vision and Image Understanding 61(3): 454-467.

Matalas, I. (1996). Segmentation Techniques Suitable for Medical Images. Electrical and Electronic Engineering Department. London, Imperial College, University of London: 321.

Morooka, K., M. Nakamoto, et al. (2013). "A survey on statistical modeling and machine learning approaches to computer assisted medical intervention: intraoperative anatomy modeling and optimization of interventional procedures." IEICE Trans. Inf. \& Syst. E96-D(4): 784-797. 
Morrone, M. C., J. Ross, et al. (1986). "Mach bands are dependent." Nature 324(6094): 250-253.

Olague, G. and R. Mohr (2002). "Optimal camera placement for accurate reconstruction." Pattern Recognition 35: 927-944.

Pentland, A. and S. Scalroff (1991). "Closed-form soultions for physically based shape modeling and recognition." IEEE Transactions on Pattern Analysis and Machine Intelligence 13: 715-729.

Pito, R. (1999). "A solution to the next best view problem for automated surface acquisition." IEEE Transaction on pattern analysis and machine intelligence 21(10): 1016-1030.

Prakoonwit, S. (2011). Twards rapid 3D reconstruction using conventional X-ray for intraoperative orthopaedic applications. Applied Signal and Image Processing Multidisciplinary Advancements. R. Qahwaji, R. Green and E. Hines. Hershey, IGI Global: 309-323.

Prakoonwit, S. and R. Benjamin (2007). "Optimal 3D surface reconstruction from a small number of conventional 2D X-ray images." Journal of X-ray Science and Technology 15(4): 197222.

Rajamani, K. T., M. A. G. Ballester, et al. (2005). A novel and stable approach to anatomical structure morphing for enhanced intraoperative 3D visualization. Proc. SPIE Medical Imaging: Visualization, Image-guided Procedures, and Display. 5744: 718-725.

Rajamani, K. T., M. Styner, et al. (2004). Bone model morphing for enhanced surgical visualization. Proc. 2004 IEEE Int. Symp. Biomedical Imaging: From Nano to Macro: 1255-1258.

Rajamani, K. T., M. A. Styner, et al. (2007). "Statistical deformable bone models for robust 3D surface extrapolation from sparse data." Medical IMage Analysis 11: 99-109.

Ritter, D., J. Orman, et al. (2007). "3D soft tissue imaging with a mobile C-arm." Computerized Medical Imaging and Graphics 31: 91-102.

Ruzon, M. A. and C. Tomasi (2001). "Edge, junction, and corner detection using color distribution." IEEE Transactions on Pattern Analysis and Machine Intelligence 23(11): 12811295.

Smith, S. M. and J. M. Brady (1997). "SUSAN - a new approach to low-level image processing." International Journal of Computer Vision 23(1): 45-78.

Staib, L. H. and J. S. Duncan (1992). "Boundary finding with parametrically deformable models." IEEE Transactions on Pattern Analysis and Machine Intelligence 17: 1061-1075.

Subsol, G., J. P. Thirion, et al. (1996). Application of an automatically built 3D morphometric brain atlas: study of cerebral ventricle shape. Visualization in Biomedical Computing, SpringerVerlag.

Szelely, R., A. Lelemen, et al. (1996). "Segmentation of 2D and 3D objects from MRI volume data using constrained elastic deformations of flexible Fourier surface models." Medical Image Analysis 1: 19-34.

Westheimer, G. (1979). "The spatial sense of the eye." Invest. Ophthalmol 18(9): 893-912. 
Zbijewski, W. and J. W. Stayman (2007). xCAT: A mobile, flat-panel volumetric X-ray CT for head and neck imaging. IEEE Nuclear Science Symposium Conference Record: 2985-2986.

Zheng, G. (2009). Statistically deformable 2D/3D registration for accurate determination of post-operative cup orientation from single standard X-ray radiograph. 12th International Conference on Medical Image Computing and Computer Assisted Intervention, London.

Zheng, G. (2010). "Statistically deformable 2D/3D registration for estimating post-operative cup orientation from a single standard AP X-ray radiograph." Annals of Biomedical Engineering 38(9): 2910-2927.

Zheng, G., M. A. G. Ballester, et al. (2006). Reconstruction of patient-specific 3D bone surface from 2D calibrated fluoroscopic images and point distribution model. International Conference on Medical Image Computing and Computer Assisted Intervention. Copenhagen, Denmark: 2532.

Zheng, G., X. Dong, et al. (2006). Robust and accurate reconstruction of patient-specific 3D surface models from sparse point sets: a sequential three-stage trimmed optimization approach. 3rd Int. Workshop Medical Imaging and Augmented Reality.

Zheng, G., X. Dong, et al. (2007). "Accurate and robust reconstruction of a surface model of the proximal femur from sparse-point data and dense-point distribution model for surgical navigation." IEEE Transactions on Biomedical Engineering 54(12): 2109-2122.

Zheng, G., S. Gollmer, et al. (2009). "A 2D/3D correspondence building method for reconstruction of a patient-specific 3D bone surface model using point distribution models and calibrated X-ray images." Medical Image Analysis 13(6): 883-899.

Zheng, G., L. P. Nolte, et al. (2011). "Scaled, patient-specific 3D vertebral model reconstruction based on 2D lateral fluoroscopy." International Jouranl of Computer Assisted Radiology and Surgery 6(3): 351-366.

Zhu, Z. and G. Li (2011). "Construction of 3D human distal femoral surface models using a 3D statistical deformable model." Journal of Biomechanics 44: 2362-2368. 